\title{
Deuteration as a Tool for Optimization of Metabolic Stability and Toxicity of Drugs
}

\author{
Sukhninder Kaur and Monika Gupta* \\ ASBASJAM College of Pharmacy, India
}

Submission: January 11, 2017; Published: March 27, 2017

*Corresponding author: Monika Gupta, ASBASJAM college of Pharmacy, India, Email: monikaguptaa@gmail.com

\begin{abstract}
Deuteration is an effective tool for the enhancement of drug's metabolic profile. Selective replacement of hydrogen with deuterium leads to increased bond strength which in turn increases the biological half-life and thus metabolic stability of the drug. Apart, deuterium substitution may also results in metabolic shunting leading to reduced exposure of vital organs to undesirable/toxic metabolites or increased exposure to desired active metabolites. This review emphasizes on various examples where deuteration came up with enhancement of metabolic stability of drug and reduction in toxicity relative to the untreated drug while retaining its significant pharmacological profile.
\end{abstract}

Keywords: Deuteration; Metabolic stability enhancement; Metabolic shunting; Kinetic isotope effect; Hydrogen-deuterium exchange; Heavy drugs

\section{Introduction}

The drug discovery process has been a very time consuming and expensive process as out of every 10,000 compounds, only one becomes a drug. Poor metabolic stability and toxicity has been the main bottle neck [1]. To overcome these issues, medicinal chemists have started investigating deuterated compounds as new chemical entities with improved properties. The idea is to selectively incorporate deuterium to alter drug metabolism and absorption properties and thus provide differentiated drugs with improved safety and/or efficacy [2]. Harold Urey, one of the most brilliant chemists of the 20th century, discovered deuterium about a year before the identification of the neutron. Deuterium occurs at a natural abundance of approximately $0.0015 \%$, which allows it to be sourced from bulk water to produce highly enriched D20 (heavy water).

Deuteration is a chemical reaction in which any covalently bonded atom is replaced by deuterium atom. The most common is hydrogen-deuterium exchange reaction. Deuterium being naturally-occurring, stable, nonradioactive isotope of hydrogen possesses similar chemical and physical properties. Thus selective deuteration has the unique effect of retaining the pharmacologic profile of drugs [3]. Deuteration can be carried out using $\mathrm{D}_{2}$ in conjugation with metal catalysts like $\mathrm{Pd}, \mathrm{Pt}, \mathrm{Rh}$ or $\mathrm{D}_{2} \mathrm{O}$ under high temperature and pressure. However, since D2 gas is extremely expensive, deuterium oxide $\left(D_{2} 0\right)$, which is relatively inexpensive and easy to obtain has been used as a heavy hydrogen source [4]. Catalytic deuteration of olefinic and acetylenic bonds is a rapid route for incorporation of deuterium [5].

From a drug development perspective, one of the most compelling characteristics of deuterium is that it forms particularly strong bonds compared with hydrogen because of greater atomic mass. A deuterium-carbon bond is almost 6 to 10 times more stable than the corresponding hydrogen-carbon bond, as a consequence is much harder to break. Thus slow rate of bond cleavage results in kinetic isotope effect (KIE) [2,6]. The kinetic isotope effect (KIE) is the relative change in the rate of a chemical reaction when one of the atoms in the reactants is substituted with one of its isotopes. Formally, it is the ratio of rate constants for the reactions involving the hydrogen $\left(\mathrm{k}_{\mathrm{H}}\right)$ and the deuterium $\left(\mathrm{k}_{\mathrm{D}}\right)$ substituted reactants [7]:

\section{$\mathrm{KIE}=\mathbf{k H} / \mathbf{k D}$}

C-D bonds having lower vibrational frequency results in lower zero-point energy than a corresponding $\mathrm{C}-\mathrm{H}$ bond which ultimately leads to higher activation energy and a slower rate (k) for C-D bond cleavage [5]. Since, the size and electronics of a deuterium atom are essentially identical to those of a hydrogen atom; the resulting deuterated compound is comparable to the hydrogen compounds with some minor shifts in physical properties such as reduced hydrophobicity, altered pKa for acids and bases. However, such differences are quiet small that effects 
of deuteration on biochemical potency or selectivity for relevant pharmacological targets are inconsiderable. Nevertheless, in certain instances, it might positively impact their metabolic fate [5].

In principle, deuterium modification has the potential to affect the biological fate of certain drugs that are metabolised by pathways involving hydrogen-carbon bond scission. For example, enzyme cytochrome $\mathrm{P}_{450}$ mediated oxidative metabolism of drugs which typically involves the cleavage of a hydrogen-carbon bond is potentially influenced by deuteration [2]. But this deuterium kinetic isotope effect (DIE) on the drug's pharmacokinetic is often masked by competing effects in-vivo, such as alternate metabolic routes and different rate-limiting steps in enzymatic reactions and biological sequestration [6]. Consequently, effect of deuteration on $\mathrm{CYP}_{450}$ metabolism is difficult to predict and vary from compound to compound and with specific deuteration pattern [2].

In addition to the effect on metabolic rate, the deuterium incorporation can sometimes also results in changes in the ratio of parent drug to metabolites and changes in the amounts of metabolites formed. It has been shown in numerous programmes that the deuterium incorporation results in altered levels of metabolites. Importantly, the metabolites formed are identical to those from the non-deuterated molecules, except for the presence of deuterium and we are not aware of deuteration resulting in unique metabolites that have not been observed for the all-hydrogen analogue. But reduced rates of metabolism and metabolic switching, where the ratio of metabolites is changed, have been reported [5].

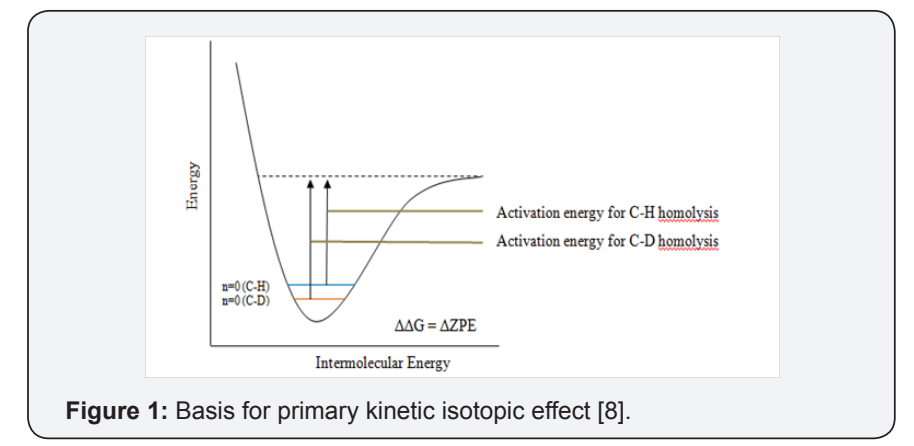

Deuterated compounds have a long history of use in humans as metabolic and pharmacokinetic probes. The first deuterated compounds being studied as potential drugs included D1Halothane, Fludalanine (2-deutero-3-fluoro-D-alanine) and $\mathrm{D}_{6}$-Nifedipine (Figure 1 \& 2) [8]. As a result, a number of deuterium substituted drugs are in clinical development. They have been widely considered in non-clinical settings and utilize as metabolic or pharmacokinetic probes clinically. But none of deuterated compound has been marketed as a human medicine till now [5]. New companies are emerging that are focussing on this area, various patents are filling with several compounds into clinical trials and altogether this developing interest in deuterated drugs ropes the possibility for valuable deuterated molecule to reach the market.

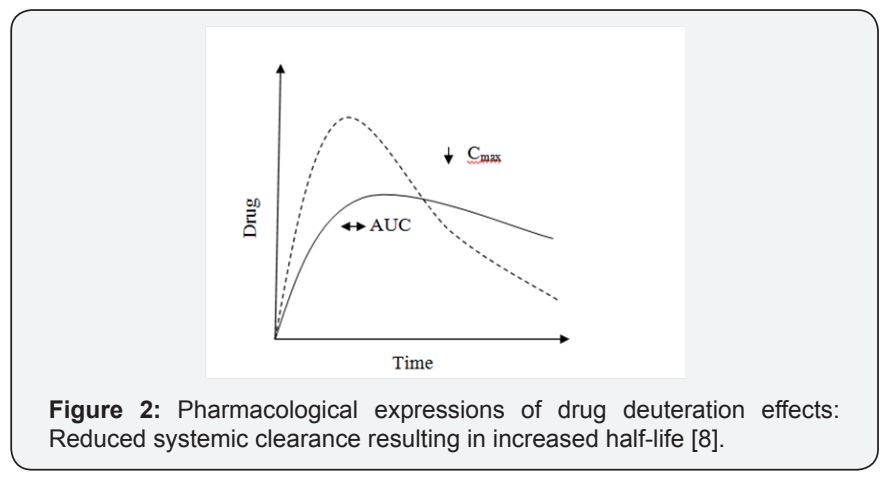

\section{Applications of Deuteration}

1. Study of reaction mechanism.

2. Elucidation of biosynthetic pathways.

3. Total synthesis: alter reaction selectivity.

4. Internal standards for mass spectrometry.

5. Enhance metabolic stability of a drug.

6. Probing metabolism related toxicity.

In the present review the potential effect of deuteration on Enhancement of metabolic stability of drug and Probing of metabolism related toxicity have been considered.

\section{Deuteration for Metabolic Stability Enhancement of Drugs}

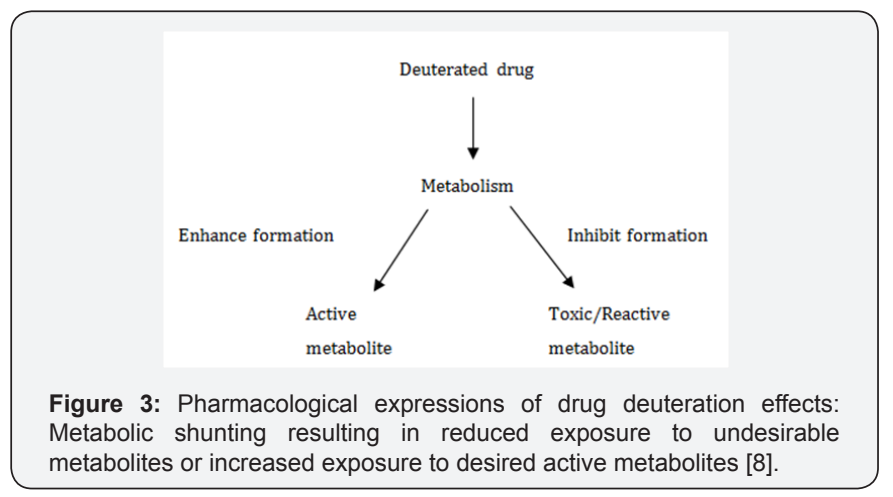

The major effect of deuteration is to reduce the rate of systemic clearance. As a result, the biological half-life of the compound is increased. As shown in (Figure $3 \& 2$ ), the duration of action of a drug increases as systemic clearance reduces. The potential clinical benefits would include the ability to maintain similar systemic exposure with increased trough levels and decreased peak levels. This could result in enhanced efficacy and lower side effects, depending on the particular drug's pharmacodynamic/pharmacokinetic relationship [5].

Following are the various preclinical and clinical deuterated agents along with some human medicines on which the effect of deuteration has been studied. 


\section{CTP-499: A deuterated pentoxifylline (Trental)}

Pentoxifylline, an active metabolite of Trental, is an effective agent for the treatment of nephropathy in Type-II diabetic patients. It is significantly metabolized to $1-((\mathrm{S})-5$ hydroxyhexyl)-3, 7-dimethylxanthine (HDX) (Structure 1), which is approved in US for the treatment of intermittent claudication and has been reported in preliminary studies to have beneficial effect on urinary albumin excretion and renal function decline. It appears that this positive pharmacology for pentoxifylline may be due in large part to the circulating metabolite (Structure 1) $[5,9]$.

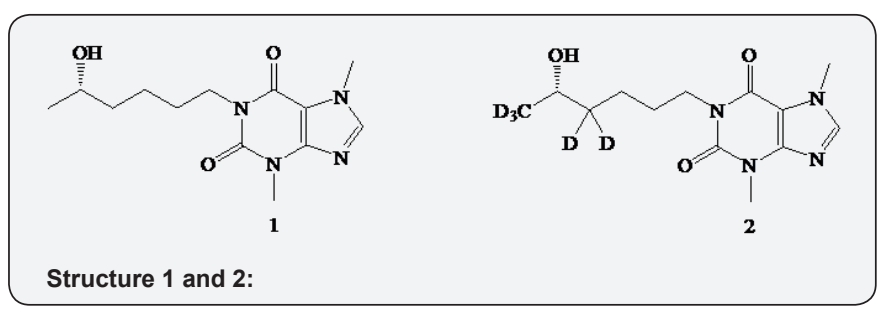

Concert Pharmaceutical has come forward with the deuterated version of (HDX) (1), i.e., CTP-499 (Structure 2), created by replacing several key hydrogen atoms with deuterium. A phase 1 clinical data with CTP-499 (Structure 2) indicated its increased exposure which ultimately leads to enhanced renoprotective effect [10]. The safety and efficacy of (Structure 2) was also studied in phase 2 clinical trials, suggesting the delay of end stage of renal failure on deuterium incorporation. Moreover, it has been showed that the deuterated drug may also act as an anti-fibrotic agent [11].

\section{Deuterated Indiplon}

Indiplon (Structure 3) is a novel sedative-hypnotic approved for the treatment of insomnia. Like other non-benzodiazepine hypnotics, its mechanism of action is to modulate subunits, especially the alpha-1 subunit, of the GABA receptor complex in order to induce sedation [12]. The deuteration leads to improvement in pharmacokinetics of Indiplon (Structure 3) by decreasing systemic clearance and in turn prolongs the half-life.

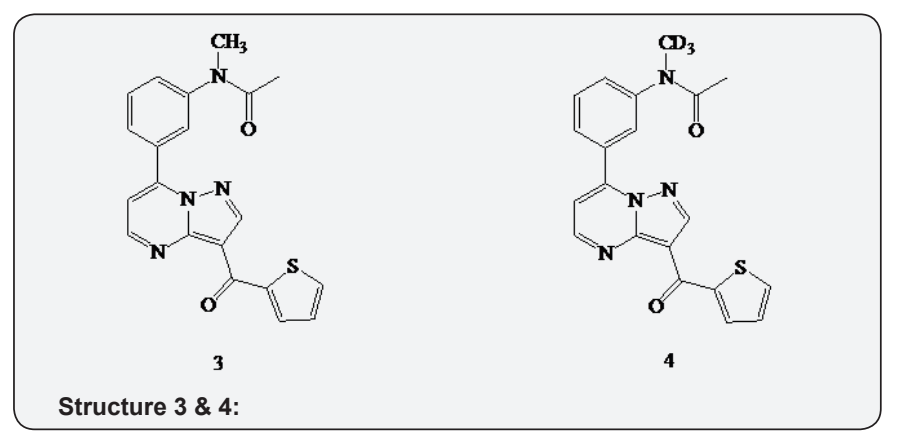

In vitro study of the deuterated analogue (Structure 4) resulted from the replacement $\mathrm{N}-\mathrm{CH} 3$ with $\mathrm{N}-\mathrm{CD} 3$, indicating the increase in half-life in both rat and human liver microsomes by $30 \%$ and $20 \%$, respectively. In-vivo study in rats showed the improvement in exposure as well as half-life by 2.6 fold and 2-fold respectively. The GABAA receptor binding affinity of both the deuterated as well as non-deuterated indiplon was found to be similar [5].

\section{CTP-354: A Deuterated L-838417}

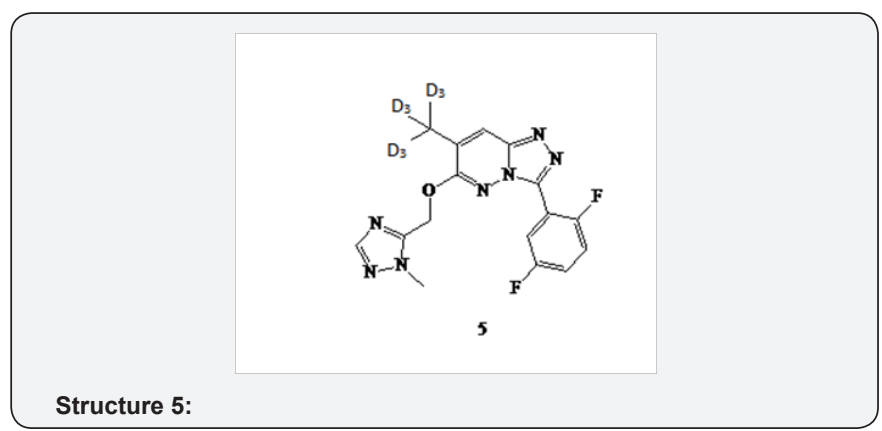

L-838417 (5), a non benzodiazepine preclinical agent, is a subtype-selective GABAA modulator developed by Merck \& Co. CTP-354 is a deuterated analogue of (Structure 5) that retains all the desirable therapeutic properties of benzodiazepines viz. - anxiolytic and spasmolytic with minimum sedative and tolerance effect. The Merck compound, L-838417 (Structure 5), was selected by Concert for deuterium substitution because its promising pharmacological profile was extensively characterised in scientific publications, yet it possessed a poor pharmacokinetic profile in preclinical testing and was never progressed into clinical development $[2,10]$. Deuterated CTP354 demonstrated substantial pharmacokinetic improvement in both In-vitro and In-vivo studies. The agent also showed longer duration of action than L-838417 (Structure 5) while possessing equivalent efficacy to gabapentin in treating the neuropathic pain $[5,10]$.

\section{C-221: A Deuterated (S)-Lenalidomide}

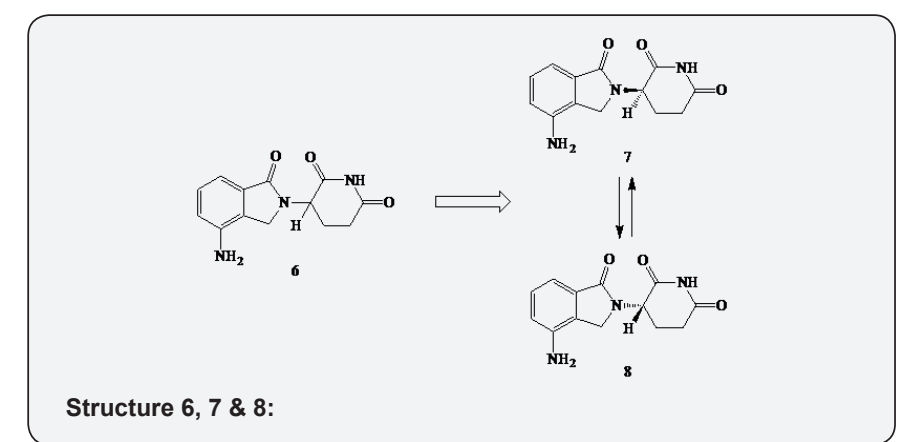

Lenalidomide (Revlimid) (Structure 6) is an antineoplastic agentindicatedfor use in multiple myelomaandin myelodysplastic syndromes (MDS). Revlimid (Structure 6) contains a mixture of $\mathrm{R}$ and S-enantiomers (Structure $7 \&$ 8) that interconvert in vivo. While the S-enantiomer (Structure 8) appears to possess the beneficial activities associated with Revlimid, dosing of the single S-enantiomer is not considered useful, as it would rapidly convert in vivo to the R/S mixture (Scheme 1). CTP-221 is a deuterium-modified S-enantiomer of the drug lenalidomide (Revlimid), developed by Concert's Pharmaceutical Inc. It has 
specific deuterium modification pattern which optimally slows the interconversion of enantiomers In-vivo, thus enabling administration of the more potent S-enantiomer with minimal exposure to the R-enantiomer. Scheme 1: In-vivo epimerization of lenalidomide enantiomers. The preclinical result suggests that CTP 221 exhibits enhanced potency compared to racemic lenalidomide, Revlimid [13].

\section{Deuterated ML-337}

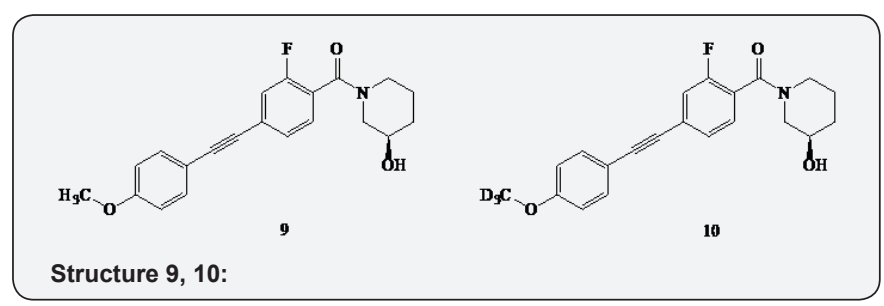

ML-337 (Structure 9) is a negative allosteric modulator at the sub-type 3 G-protein-coupled metabotropic glutamate receptors (mGluRs). The mGluRs have emerged as new drug targets with potential for treatment of a range of CNS disorders. Numerous studies indicate that mGlu3 is the key mGluR subtype involved in glialneuronal communication, and inhibition of mGlu3 is hypothesized to have therapeutic utility in the treatment of cognitive disorders, schizophrenia, depression and Alzheimer's disease [14]. The structure-activity relationships revealed that p-OCH3 substituent was required for the desired pharmacology. However, the CYP mediated demethylation has been identified as a major metabolic pathway. As a consequence, the author synthesized the deuterated methoxy compound (Structure 10) in order to have improved metabolic stability. In vitro as well as in vivo studies revealed the improvement in metabolic stability on deuteration by showing slower plasma clearance $[5,14]$.

\section{Deuterated Odanacatib}

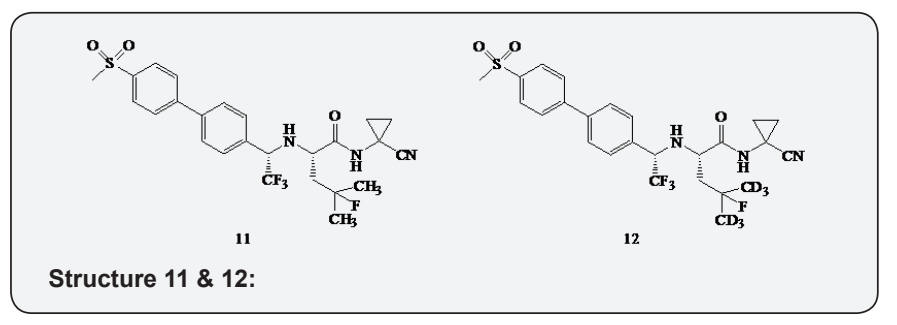

Odanacatib (Structure 11) is a cathepsin K inhibitor for the treatment of post menopausal osteoporosis. Cathepsin $\mathrm{K}$ is the primary enzyme involved in osteoclast mediated bone resorption. The agent is under phase 3 clinical trials and studies have indicated that hydroxylation of the fluoro-isobutyl side chain is the major metabolic pathway (Scheme 2). The deuterated analogue (Structure 12) prepared was resulted in improved pharmacokinetic profile such as longer half-life and greater exposure [5].

\section{AVP-786: A Combination of Deuterated Dextromethorphan and Quinidine.}

A fixed-dose combination of dextromethorphan hydrobromide and quinidine sulfate $(20 \mathrm{mg} / 10 \mathrm{mg}$ ) is currently marketed by Avanir with the name Nuedexta. It is used for the treatment of Pseudo bulbar Affect (PBA) [5], a neurological condition resulting in sudden and exaggerated episodes of laughing or crying. AVP-786 is a combination of a deuterated dextromethorphan and ultra-low dose quinidine that is planned for clinical development by Avanir Pharmaceuticals in treatment-resistant major depressive disorder [5,15]. Another combination of dextromethorphan and quinidine, referred to as AVP-923, are also in clinical development for other neurologic indications, including agitation in Alzheimer's disease and levodopa-induced dyskinesia. The quinidine in both Nuedexta and AVP-923 inhibits CYP2D6, the major metabolic pathway in humans that converts dextromethorphan (Structure $13 \& 14$ ) to dextrorphan (Structure 15) (Scheme 3). Thus quinidine acts as a pharmacokinetic booster.

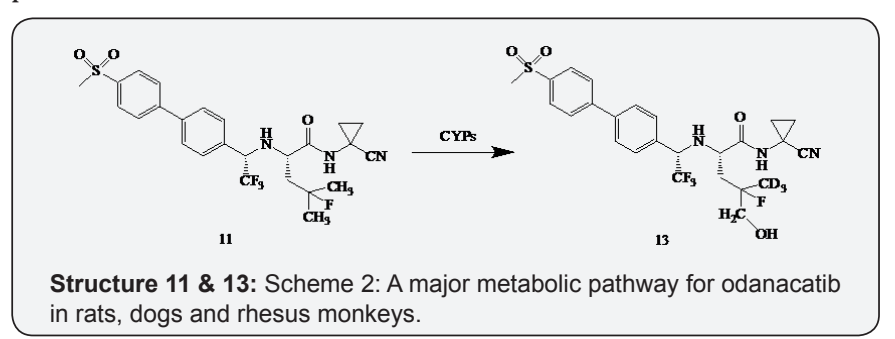

The deuterated dextromethorphan in AVP-786 maintains the pharmacologic profile of dextromethorphan but is less susceptible to CYP2D6 catalyzed metabolism due to the deuterium isotope effect. In a Phase 1 trial comparing AVP-923 to AVP-786, which has a substantially lower dose of quinidine but equivalent doses of dextromethorphan and deuterated dextromethorphan, AVP-786 provided essentially identical plasma levels of deuterated dextromethorphan compared to (nondeuterated) dextromethorphan from AVP-923 [5,16].

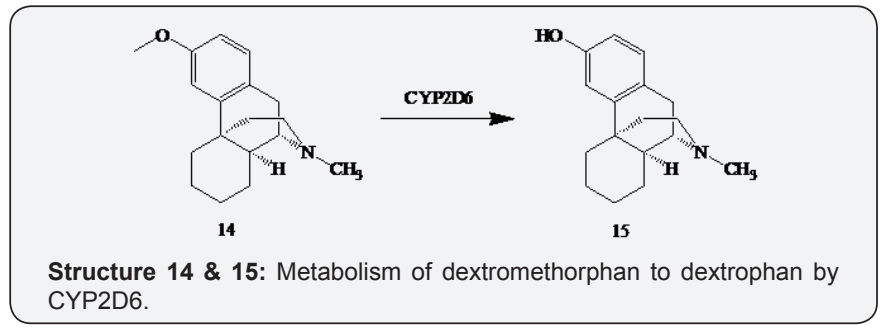

\section{C-10068: A Deuterated dextroethorphan}

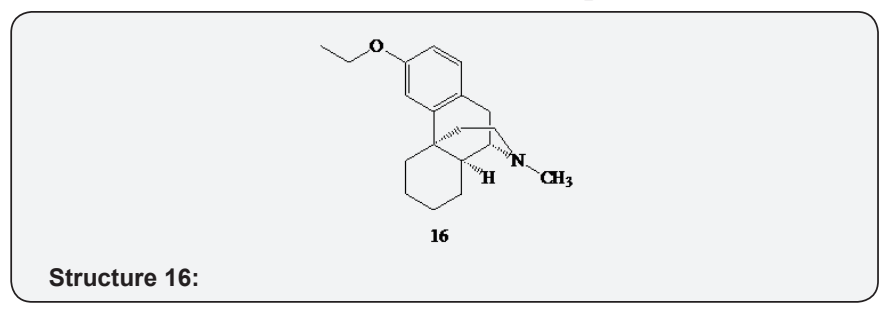

Dextroethorphan (Structure 16) is an agent which is closely related to dextromethorphan (Structure 14), an anti-tussive agent and active ingredient in Nuedexta. C-10068 (Structure 17) is a selectively deuterium modified analogue of dextroethorphan (Structure 16). Research conducted by the Walter Reed Army Institute of Research (WRAIR) showed that this novel 
deuterium-containing sigma-1 agonist, C-10068 (Structure 17), invented at Concert, has anti-seizure and anti-inflammatory effects in a preclinical model of traumatic brain injury (TBI). The drug has complex pharmacological actions at several neurologically relevant receptors which appear generally similar to those of parent dextromethorphan (Structure 14) although it binds strongly to the sigma-1 receptor. C-10068 (Structure 17) has shown anticonvulsant properties and evidence of neuroprotective, antinociceptive and antidepressant effects. Hence it has potential as a therapeutic agent for the treatment of pain, depression, seizures and traumatic brain injury. C-10068 (Structure 17) was studied for metabolic stability in human liver microsomes (HLM) and CYP2D6 supersomes and compared with that of dextroethorphan (Structure 16) and dextromethorphan (Structure 14). The CYP450 profile of the compound has suggested that it is a deuterated new chemical entity that has superior metabolism and pharmacokinetic properties compared to (Structure 16 and 14) [17].

\section{Deuterated Teleprevir}
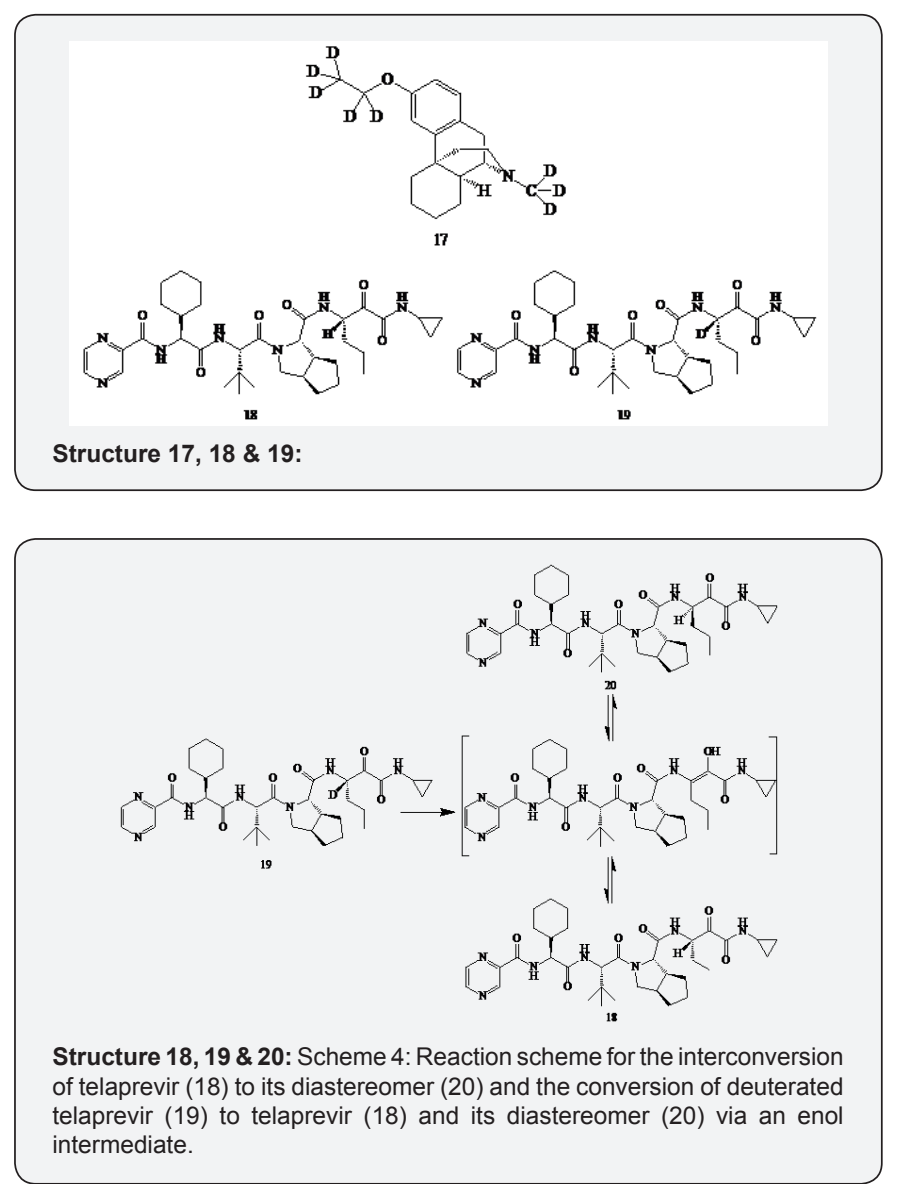

Telaprevir (Structure 18) is an inhibitor of hepatitis $C$ viral NS3-4A protease that was approved as Incivek in 2011 for the treatment of hepatitis C infection. Telaprevir (Structure 18) is the S-diastereomer in which the chiral center adjacent to the ketoamide moiety has the S-chirality. As shown in Scheme 4, (Structure 18) epimerizes In-vivo via an enol intermediate to the
R-diasteromer (Structure 19,20), which is the major circulating metabolite in plasma and is a 30 -fold less active protease inhibitor than (Structure 18). The hydrogen at this chiral centre was replaced with deuterium to provide deuterated telaprevir (Structure 19), which should slow the rate of conversion to the enol and the resulting epimerization to the R-diastereomer (Structure 20) [5].

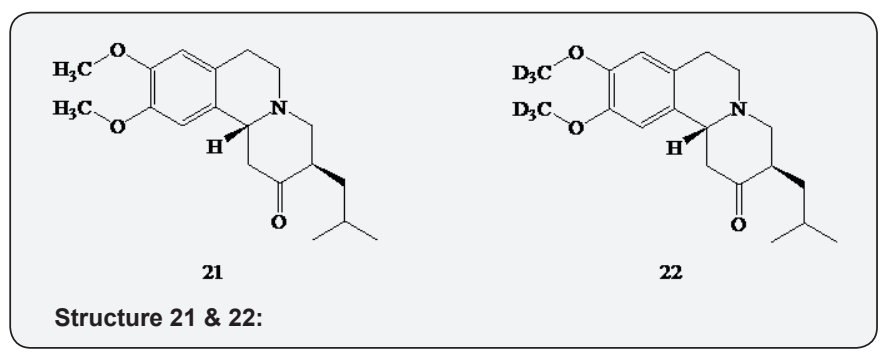

\section{SD-809: A Deuterated Tetrabenazine}

Tetrabenazine (Structure 21) is the only FDA-approved treatment for chorea associated with Huntington's disease. A deuterated version of Tetrabenazine (Structure 21) is SD-809 (Structure 22) which is developed by Auspex Pharmaceuticals and is currently in a Phase 3 registration trial for the treatment of the same [5,17]. After oral dosing of Tetrabenazine (Structure 21) or SD-809 (Structure 22), the compounds undergo extensive and rapid metabolism to the major circulating active metabolites (Scheme 5). The active metabolites (Structure 23) are then further metabolized by CYP2D6 to (Structure 24) and (Structure 25) (Scheme 5).

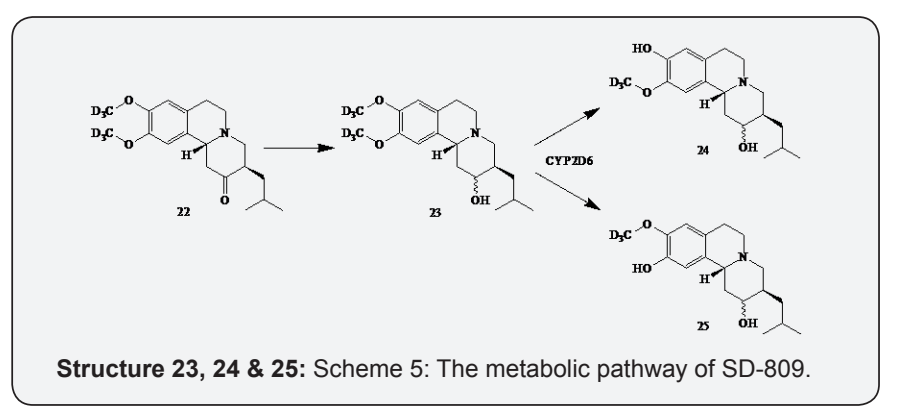

Tetrabenazine (Structure 21) is associated with high rates of adverse events, which include sedation/somnolence, fatigue, insomnia, depression, akathisia, and nausea that may be associated with not only the Cmax levels of the parent but also the variable levels of active metabolites due to inter-patient variability in CYP2D6 activity. Thus its dosing requires careful titration to identify a dose (up to three-times daily) that reduces chorea and minimizes the adverse events mentioned above. The pharmacokinetic profile may allow SD-809 (Structure 22) to achieve similar exposures with a lower Cmax at a lower dose than tetrabenazine [5].

\section{CTP-518: A deuterated atazanavir}

Atazanavir (Structure 26) is an antiretroviral drug of the protease inhibitor class. It is used to treat infection of human 
immunodeficiency virus (HIV). The current standard of care is to co-administer all marketed HIV protease inhibitors with ritonavir, a pharmacokinetic booster, to increase the blood levels of these antiretroviral drugs. However, ritonavir co-dosing complicates dosing regimens and is associated with nausea and increased blood lipids, including LDL cholesterol and triglycerides. Concert recently announced initiation of multiple ascending dose studies with its development candidate CTP518 (Structure 27), an HIV protease inhibitor (PI) based on the atazanavir (Structure 26) scaffold. In preclinical studies, the antiviral potency of (Structure 26) was fully retained but with markedly slower hepatic metabolism, providing an increase in half life and plasma trough levels. Due to favourable antiviral potency and pharmacokinetic profile, CTP-518 (Structure 27) is being developed to be optimally administered without the need for ritonavir. Eliminating the need for this agent is clinically advantageous $[6,18]$.

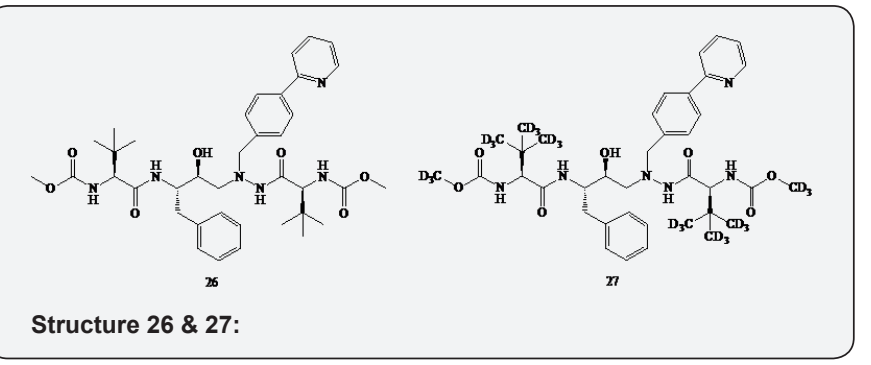

\section{SD-254: A Deuterated Venlafaxine}

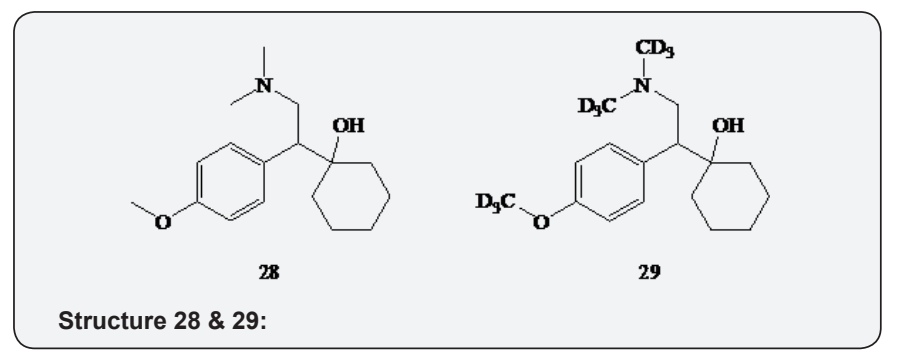

Venlafaxine (Structure 28) (Effexor) is an antidepressant of the serotonin-norepinephrine reuptake inhibitor (SNRI) class [19-21]. It is subject to 0-demethylation as the major metabolic pathway, with $\mathrm{N}$-demethylation playing a secondary role, and is a substrate of the polymorphic enzymes CYP2D6 and 2C19 [22]. Auspex Pharmaceuticals is developing SD-254 (Structure 29), a selectively deuterated analogue of the Wyeth's top-selling anti-depression drug venlafaxine (Structure 28). In October 2008, Auspex issued a press release on their SD-254 (Structure 29) Phase I healthy volunteer study in which the compound reportedly "exhibited a pharmacokinetic profile that appears to be superior to that of venlafaxine (Structure 28)" [6] and early clinical studies indicated increased exposure of the parent drug, reduced exposure of the 0 -demethyl metabolite with less variability in the ratio of the 0 -demethyl metabolite to parent drug [23]. Hopefully, the agent (Structure 29) could be the first drug that combines the effectiveness of the so called serotonin norepinephrine reuptake inhibitor (SNRI) class of depression drugs but with the improved side effect profile of the selective serotonin reuptake inhibitor (SSRI) class - potentially making it very attractive for first line use [24].

\section{BDD-10103: A Deuterated Tolperisone}

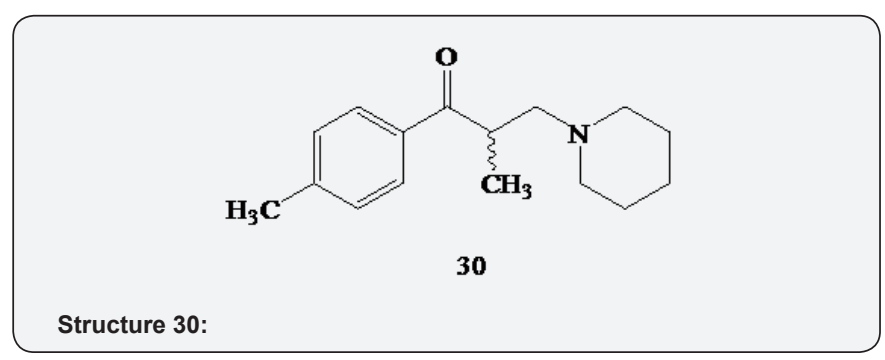

Tolperisone (Structure 30), a piperidine derivative, is a centrally acting muscle relaxant. It has various advantageous features including a low adverse event profile, a lack of sedation and no interaction with alcohol as well as no potential for tolerance and addiction. However, tolperisone (Structure 30), which nowadays is generic compound, has several pharmacological limitations, mainly a high first pass effect in various species which requires high daily doses for compensation and a metabolism which is dependent on the genetic background of the patients and thus open for a considerable inter-individual pharmacokinetic variation. Berolina innovative Research and Development Services Pharma (BiRDS Pharma) is developing BDD-10103, a deuterated analogue of tolperisone (Structure 30) [25]. BDD-10103 addresses the drawbacks of tolperisone by simultaneously keeping all its advantageous features. Various animal models confirmed that oral doses of BDD-10103 are more potent than the corresponding doses of tolperisone (Structure 30 ) both in selected preclinical rodent and non-rodent animal models: BDD-10103 and tolperisone (Structure 30) inhibited the monosynaptic ventral root reflex roughly to the same extent. Metabolic degradation of BDD-10103 and (Structure 30) indicated the longer elimination half life for BDD-10103 in man.

BDD-10103 follows a typical CYP2D6, CYP2C19 metabolism as does tolperisone. The compound is however more stable against biodegradation by liver enzymes of the CYP family. Interestingly, formation of certain metabolites that occurred in the course of plain tolperisone biodegradation is suppressed within the BDD-10103 metabolism and an enhanced overall bioavailability of BDD-10103 has been observed [26].

\section{Deuteration for Probing Metabolism Related Toxicity}

An extension of the use of deuterium isotope effects to enhance metabolic stability of the drug is their use to probe mechanisms of the formation of reactive metabolites that can cause toxicity. Drugs and other chemicals are usually eliminated from the body by multiple pathways, and deuterium substitution for hydrogen at sites of metabolism may decrease, increase, or have no effect on the toxicity of a compound [27]. Many drugs are metabolized in complex patterns, resulting in the formation of both active and innocuous metabolites in addition to reactive 
or toxic metabolites. (Structure 3) illustrates metabolic shunting in which deuterium substitution reduces the formation of an undesired or toxic metabolite as well as enhancing the formation of a desired metabolite [5].

It should be emphasized that the magnitude of a deuterium isotope effect on toxicity is dependent on several variables that can affect both the enzyme and transporters involved in metabolism and disposition of the compound and its metabolites, or the response of the cell, tissue, or animal to the tissue damage. Furthermore, dose-response studies are important, since there often are dose-thresholds for toxicity so that above a certain dose, enough reactive metabolite may be formed, even with the deuterated compound, to cause toxicity equivalent to that of the protiated compound [27].

The potential benefits of metabolic shunting in terms of human medicines will be discussed for following examples:

\section{Nitrosamines and Related Compounds.}

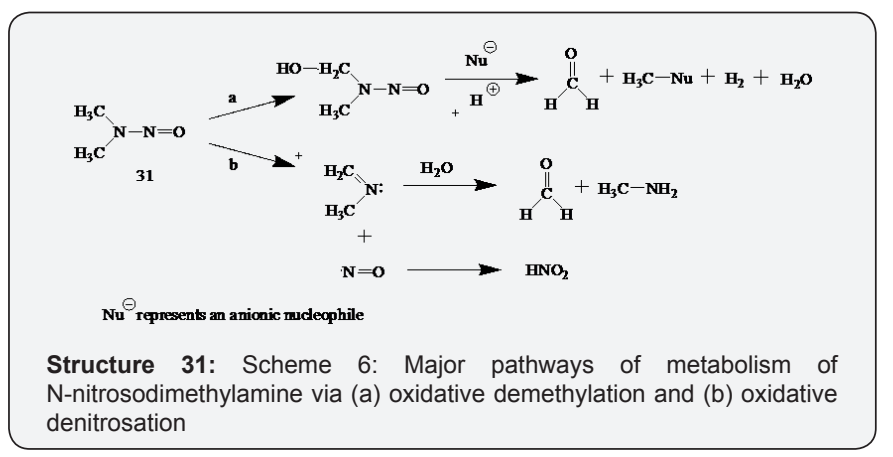

N-Nitrosodimethylamine (NDMA) (Structure 31) is a hepatocarcinogen in rats, and Keefer et al. (1973) found that overall tumor incidence in NDMA-treated rats is much greater than in NDMA-d6-treated rats. At 5-fold higher doses, 18 hepatomas were detected in the NDMA-d6-treated group and 24 hepatomas in the NDMA-treated group [27-29]. The two major pathways of oxidation of (NDMA) (Structure 31) are described in scheme 6. Pathway leads to the generation of the DNA-methylating agent, whereas pathway b generates methylamine, formaldehyde and nitrite. Streeter have observed an approximately 3 -fold increase in the denitrosation pathway after NDMA-d6 administration to rats compared with NDMA. For this to occur, either enzymes other than CYP2E1 can cause denitrosation of NDMA, or a switch in mechanism in the denitrosation of NDMA takes place upon deuteration $[27,30]$.

In contrast to the results with nitrosamines, substitution of deuterium for hydrogen on carbons $\alpha$ to nitrosoureas, nitrosourethanes, and nitrosoguanidines has little or no effect on mutagenicity [27,31,32] or carcinogenicity [33]. However, deuterium substitution in some of these structures, such as the antitumor agent 1,3-bis (2-chloroethyl)-1-nitrosourea, has revealed multiple mechanisms of elimination and hydrolysis in generating reactive metabolites $[27,34,35]$.

\section{Procarbazine}

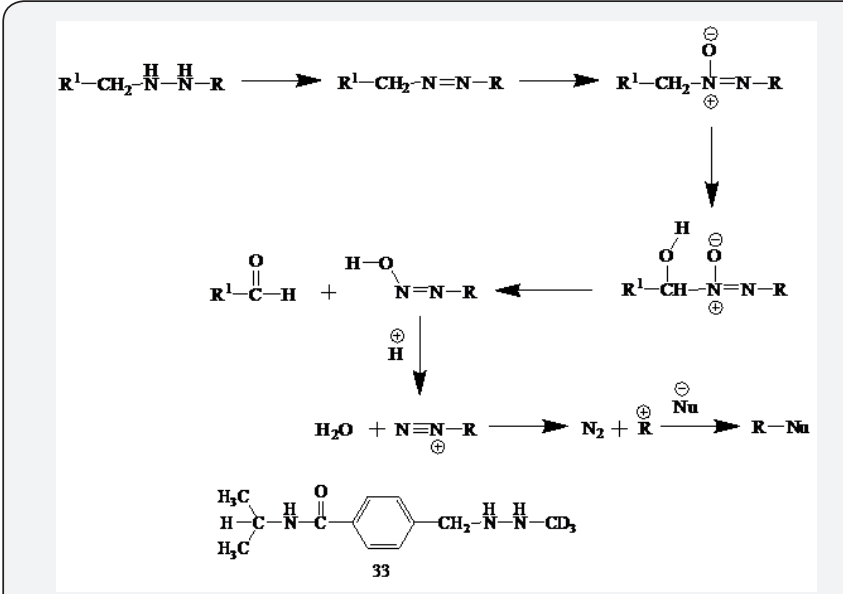

Structure 33: Scheme 7: Major pathway of oxidation of 1, 2-disubstituted hydrazines to reactive alkylating metabolites

The antitumor drug, procarbazine (Structure 32), is a 1, 2-disubstituted hydrazine that is metabolized to reactive metabolites like other such hydrazines (Scheme 7) [27,36,37]. In humans procarbazine causes testicular damage leading to azoospermia [27,38]. It caused a similar sterility in mice that was reversed by deuterium substitution for hydrogen at the benzylic carbon atom (Structure 33), but not on the N-methyl group (Structure 34) [27,39]. Consistent with these results, a deuterium isotope effect $(\sim 4)$ was observed on genotoxicity in rat liver and testes after administration of either procarbazine or its N-CD3 analogue versus its CD2-benzylic analogue $[27,40]$. The results suggest that oxidative debenzylation occurs with concomitant formation of either methyldiazene or N1-hydroxyN2-methyldiazene as the toxic or proximate toxic species (Scheme 7) [27].

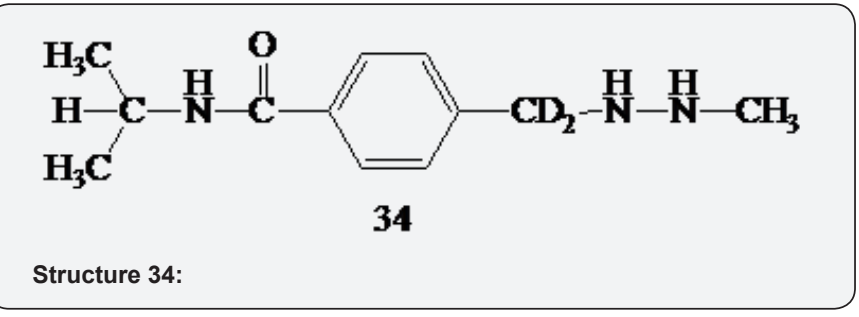

Deuterated Tamoxifen

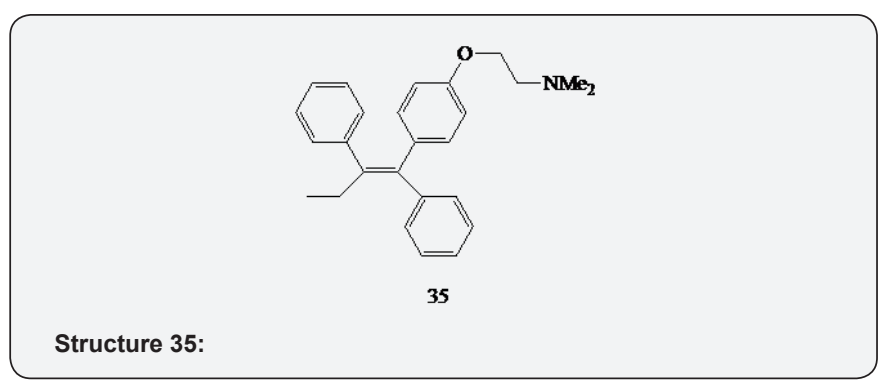

The nonsteroidal antiestrogen tamoxifen (Structure 35) has been used as an adjunct chemotherapeutic agent for the treatment of all stages of breast cancer. However, tamoxifen has 


\section{Global Journal of Pharmacy \& Pharmaceutical Sciences}

been reported to increase the risk of endometrial cancer and acted as a liver carcinogen in rats. Tamoxifen (Structure 35) is known to be both an estrogen antagonist and agonist, hence like other estrogens; it has been shown to be a hepatic tumor promoter in rats. It has also been observed that tamoxifen is metabolized to derivatives capable of binding to DNA. Thus, tamoxifen can act as a complete carcinogen, by initiating tumors through DNA adduct formation and promoting tumors through its estrogenic activity. Metabolic pathway proposed for the activation of tamoxifen to a reactive electrophile involves allylic $\alpha$-carbon oxidation that may generate a reactive quinone methide.

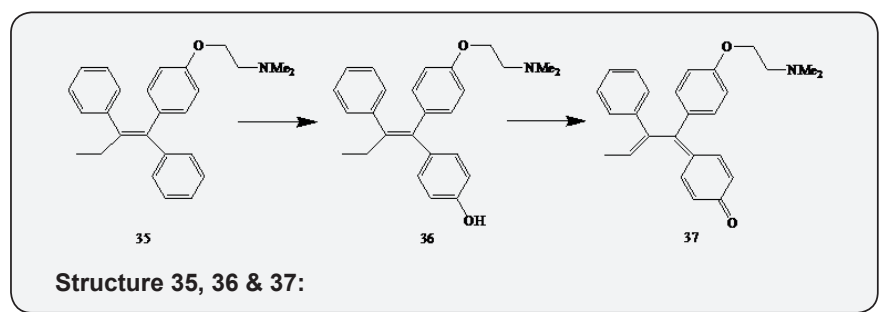

Oxidation of one of the phenyl rings give 4-hydroxytamoxifen (Structure 36), which may undergo a subsequent oxidation to 4-hydroxytamoxifen quinone methide (Structure 37) (Scheme 8), an electrophile that can yield DNA adducts through Michaeltype addition reactions [41]. Genotoxicity of this widely used antitumor drug was decreased 2 to 3 fold in-vivo in rats and in vitro in a MCL-5 human cell line that retains cytochrome P450 activity by using d5-tamoxifen (Structure 38) in which deuterium is substituted for hydrogen in the allylic ethyl group. These and other results suggest that liver carcinogenicity in rats caused by tamoxifen involves allylic $\alpha$-carbon oxidation that may generate a reactive quinone methide $[27,42]$.

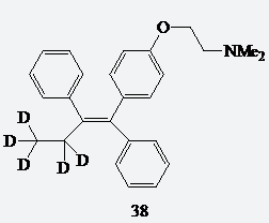

Structure 38: Scheme 8: Proposed metabolic activation pathways for tamoxifen

\section{Deuterated Nevirapine}
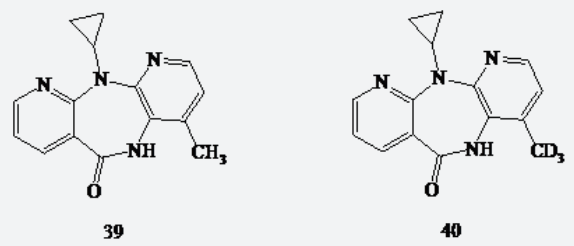

Structure $39 \& 40$ :

Nevirapine (Structure 39) provides an example of metabolite shunting in which a deuterated analogue (Structure 40) was prepared that showed effects upon both the metabolic clearance and toxicity. Nevirapine (Structure 39) is a non-nucleoside reverse transcriptase inhibitor for the treatment of HIV infection that is associated with a relatively high incidence of skin rash and hepatotoxicity in humans. The proposed pathway to the hepatotoxicity and skin rash by the drug is due to production of radical intermediate during CYP metabolism (Scheme 9). The radical intermediate produced can either lose a hydrogen atom to produce reactive metabolite (Structure 41) or undergo hydroxylation to metabolite (Structure 42), which can be sulfated to reactive metabolite (Structure 43). The deuterium substitution (Structure 40) significantly reduces (approx. 5-fold) covalent binding to hepatic proteins in both mouse and rat hepatic microsomes. It also resulted in decreased covalent binding with CYP3A4 supersomes.

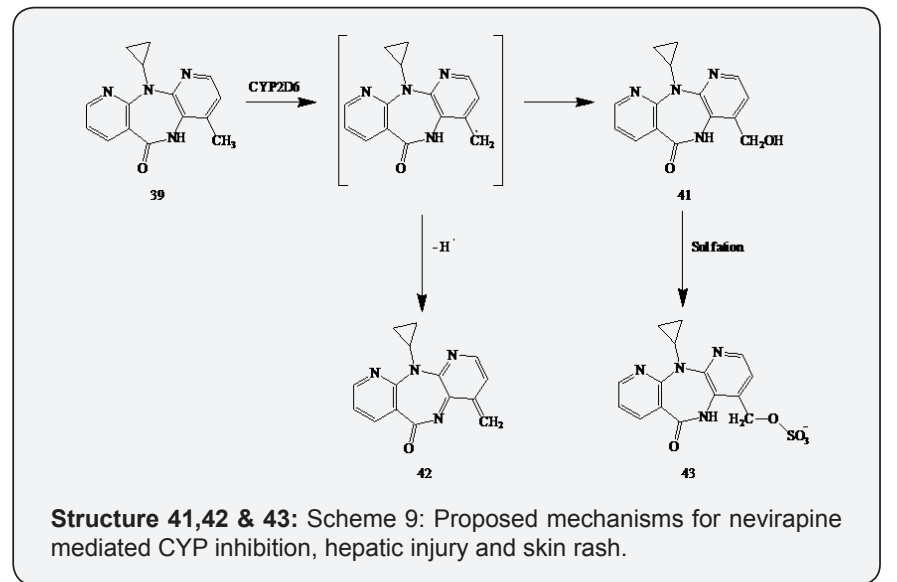

These data support the formation of reactive intermediate (Structure 42) that not only inactivates CYP metabolic enzymes but may also produce the hepatotoxicity of nevirapine (Structure 39) After oral dosing in rats, deuterated nevirapine (Structure 40) was rapidly cleared, resulting in plasma levels that were much lower than observed with the same dose of nevirapine (Structure 39). This increase in clearance for deuterated analogue (Structure 40) is attributed to metabolic switching away from the postulated reactive metabolite (Structure 42 ) that inactivates CYP450, which results in less CYP inhibition and faster in vivo clearance. It has also been observed that nevirapine (Structure 39) is oxidized by CYP enzymes to the 12-hydroxy metabolite (Structure 41), which is then activated by sulfotransferases in the skin to the sulfate ester (Structure 43). This sulfate ester may be the metabolite that covalently modifies skin proteins thereby resulting in an immune-mediated rash. As the deuterated agent (40) produced much less 12-hydroxy-metabolite (Structure 41), leading to reduction in the incidence and severity of the rash [5].

\section{Deuterated Phenacetin}

Phenacetin (Structure 44) has been widely used as a painrelieving and fever-reducing drug, until banned in the US by the FDA in 1983. Its use has declined because of its adverse effects, which include increased risk of certain cancers and kidney damage. It is metabolized into paracetamol, which replaced it in some over-the-counter medications following the ban [43]. Whereas deuterium substitution for hydrogen in the ethoxymethylene carbon of phenacetin [d2-phenacetin 
(Structure 45 ) significantly decreases ( $\sim 3$ fold) the incidence and extent of hepatic necrosis in hamsters, however, it significantly increased blood methemoglobin concentrations [27,44].

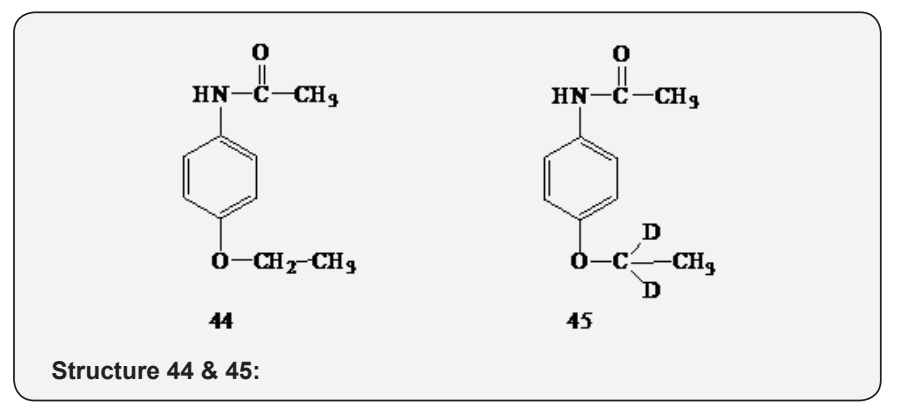

A deuterium isotope effect $(\sim 2)$ was observed for hamster liver microsomal oxidation of phenacetin (Structure 44) to acetaminophen (Structure 46) (Scheme 10), which is further oxidized to its reactive toxic quinine imine (Structure 47) the major proximate hepatotoxic metabolite of phenacetin in hamsters. Recent investigations with human CYP1A2 and some mutants revealed similar inter- and intramolecular deuterium isotope effects $(\sim 2-3)$ for oxidative 0-deethylation of phenacetin, to acetaminophen, indicating that $\mathrm{C}-\mathrm{H}$ bond cleavage is only partially rate-determining in the process $[27,45]$. Increased concentrations of methemoglobin observed with deuterated phenacetin analogs suggest that there is a shift in metabolism from oxidative 0-deethylation to hydrolysis to p-phenetidine (Structure 48) (Scheme 10), which is known to be oxidized to products that can cause methemoglobinemia and hemolysis $[26,46]$.

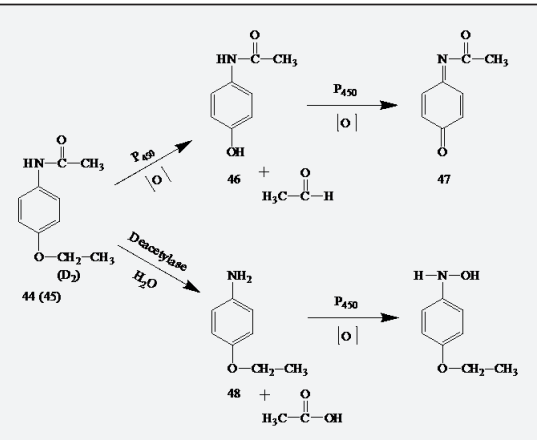

Structure 46,47 \& 48: Scheme 10: Partial scheme for the metabolism of phenacetin via oxidative O-deethylation to acetaminophen, which is further oxidized to its reactive toxic quinine imine metabolite, and via amide hydrolysis to $\mathrm{p}$-phenetidine, which can be further oxidized to a hydroxylamine that can itself, or via its nitroso oxidation product, cause methemoglobinemia.

\section{CTP-347: Deuterium Modification of Paroxetine}

Paroxetine (49) is a centrally acting SSRI (selective serotonin reuptake inhibitor) for the treatment of major depressive disorder, panic disorder, social anxiety disorder, and premenstrual dysphoric disorder. It had good efficacy in treating hot flashes. Paroxetine (Structure 49) was found to be metabolized by CYP2D6 by the proposed mechanism shown in (Scheme 11). However, it was also found that it inhibited its own metabolism by irreversibly inactivating the enzyme. Its use, therefore, can be complicated in patients potentially benefiting from this agent due to possible drug-drug interactions with other drugs metabolized by CYP2D6. In the case of thioridazine, co administration of paroxetine (Structure 49) is contraindicated. The drug-drug interactions observed for paroxetine (Structure 49) are believed to be predominantly due to irreversible inactivation of CYP2D6 [5,10].

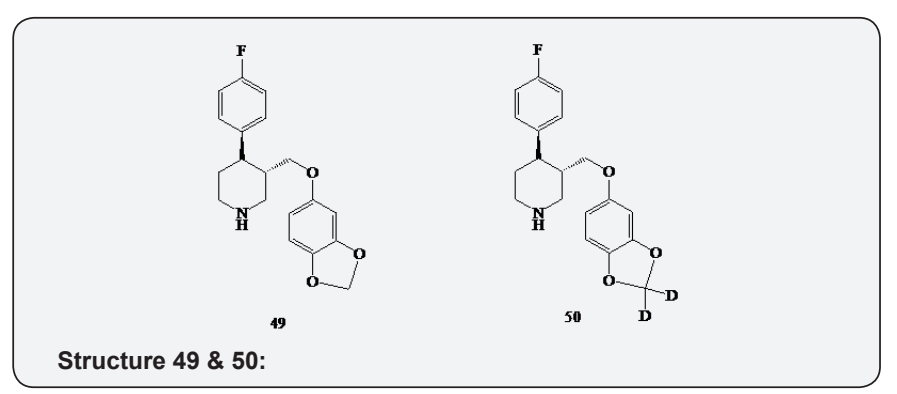

Concert Pharmaceutical's CTP-347 (Structure 50) is a selectively deuterated analogue of paroxetine (Structure 49). CTP-347 was designed to eliminate the irreversible inhibition of a key metabolizing enzyme (CYP2D6) caused by a highly reactive paroxetine metabolite that covalently binds to the enzyme [10]. CTP-347 (Structure 50) demonstrated little or no CYP2D6 inactivation, presumably due to metabolic shunting away from Pathway A to Pathway B, thereby favouring the ring opening pathway to yield innocuous metabolites [5]. Moreover, the intrinsic pharmacology of paroxetine (Structure 49) was unchanged after deuterium incorporation [10]. This deuteration is the first clinical demonstration where the deuteration can be utilised to ameliorate drug-drug interactions in humans [6]. Other examples of drugs on which deuteration was done include rofecoxib, fludalanine, $\mathrm{N}$-methylformamide and chloroform.

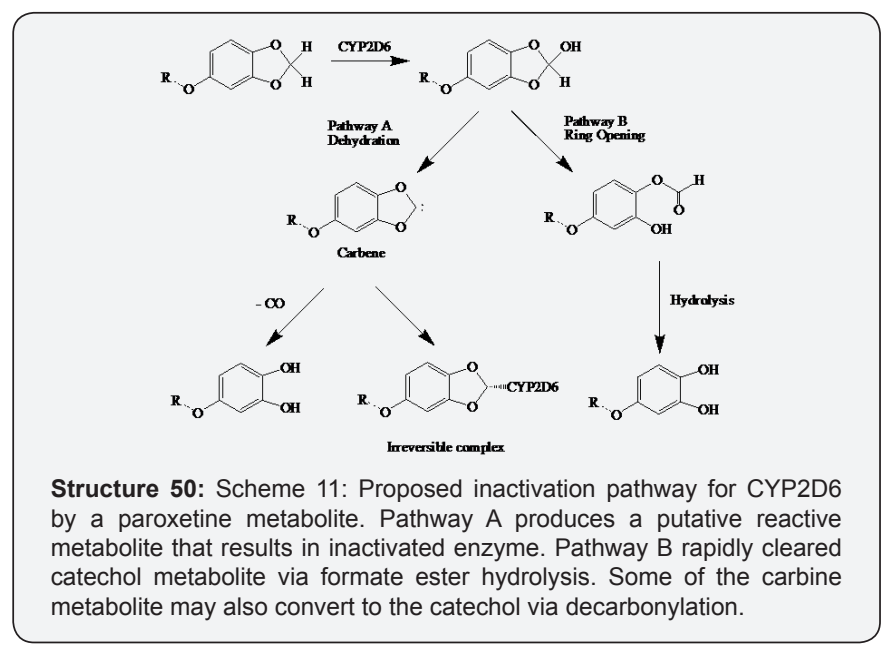

\section{Conclusion}

The authors conclude that Deuterium incorporation is an easy technique to initiate. Deuterium kinetic isotope effect leads to reduction in the rate of systemic clearance leading to prolongation of the biological half-life. On the other hand, in case of formation of toxic metabolites, deuteration results in 


\section{Global Journal of Pharmacy \& Pharmaceutical Sciences}

metabolic shunting where formation of toxic metabolites was reduced. There has also been a much greater understanding of the complexity and unpredictability of the effects of deuterium replacement upon pharmacokinetics and metabolism. Improving the pharmacokinetic and/or toxicological properties of existing drugs by deuteration continues to show significant potential. Much failure of medicinal agents in clinical trials involves three main reasons: lack of efficacy, toxicity or poor pharmacokinetics: deuterated drugs benefit from avoiding the first, while they can specifically address the last two. Since much of the groundwork for the trials has been performed upon the non-deuterated versions, there is great hope that this will enable faster, smarter and cheaper trials of the deuterated version. So the advancement of the deuterated compounds in clinical trials is highly promising.

\section{References}

1. Veljkovic V, Veljkovic N, Esté JA, Hüther A, Dietrich U (2007) Application of the EIIP/ISM bioinformatics concept in development of new drugs. Curr Med Chem 14(4): 441-453.

2. Kristen C Buteau (2009) Deuterated Drugs; unexpectedly nonobvious?. J High Tech L 10(1): 22-74.

3. Vidal JB, Alonso DJA, Buil AMA, Eastwood PR, Esteve TC, et al. (2013) New crth2 antagonists. Patent WO2013010880 A.

4. Kosaku Hirota, Hironao Sajiki, Nobuhiro Ito (2008) Method for producing deuterium gas and catalytic deuteration method using deuterium gas obtained thereby. U. S. patent 20080145303.

5. Scott L Harbeson, Roger D Tung (2014) Deuterium medicinal chemistry: A new approach to drug discovery and development. Med Chem. News 8-22.

6. Bell RP (1974) Recent advances in the study of the kinetic hydrogen isotopic effects. Chem Soc Rev 3: 513-544.

7. Kensei Kobayashi (2015) Kinetic isotope effect. Encyclopedia of Astrobiology pp1337.

8. Gant TG (2014) Using Deuterium in Drug Discovery: Leaving the Label in the Drug, J Med Chem 57 (9): 3595-3611.

9. Wesheimer FH (1961) The magnitude of the primary kinetic isotope effect for compounds of hydrogen and deuterium. Chem Rev 61(3): 265-273.

10. Graham P, Liu J, Turnquist D (2012) Deuterium modification as a new branch of medicinal chemistry to develop novel, highly differentiated drugs. Drug Dev Deliv 12(6): 67-70.

11. Concert Pharmaceuticals Inc. (2014) Concert Pharmaceuticals presents positive 48-week results from Phase 2 clinical trial of CTP499 in diabetic kidney disease. Press Release.

12. Lankford A (2007) Indiplon in the treatment of sleep disorders. Neuropsychiatr Dis Treat 3(6): 765-773.

13. Concert Pharmaceuticals, Inc. (2012) Concert Pharmaceuticals presents findings demonstrating enhanced potency of CTP-221, a deuterated S-enantiomer of lenalidomide. Press Release.

14. Wenthur CJ, Morrison R, Felts AS, Smith KA, Engers JL, et al. (2013) Discovery of (R)-(2-fluoro-4-((-4-methoxyphenyl)ethynyl)phenyl) (3-hydroxypiperidin-1-yl) methanone (ML337), an mGlu3 selective and CNS penetrant negative allosteric modulator (NAM). J Med Chem 56(12): 5208-5212.
15. Avanir Pharmaceuticals, Inc. (2014) Avanir Pharmaceuticals reports fiscal 2014 first quarter financial and business results.

16. Avanir Pharmaceuticals, Inc. (2013) Avanir Pharmaceuticals announces positive interim data from pharmacokinetic study with next generation compound AVP-786.

17. Concert Pharmaceuticals, Inc. (2011) Concert Pharmaceuticals presents preclinical data on novel deuterium-containing drug compound at antiepileptic drug trials XI conference; sigma- 1 agonist, C-10068, shown to have anti-convulsant and neuroprotective effects.

18. Auspex Pharmaceuticals, Inc. (2013) Auspex initiates Phase 3 trial of SD-809 in Huntington's Disease Press Release.

19. Concert Pharmaceuticals, Inc (2009) Concert Pharmaceuticals initiates Phase $1 \mathrm{~b}$ clinical trial of CTP-518, protease inhibitor for treatment of HIV" Press Release.

20. Muth EA, Haskins JT, Moyer JA, Husbands GE, Nielsen ST (1986) Antidepressant biochemical profile of the novel bicyclic compound Wy-45,030, an ethyl cyclohexanol derivative. Biochem Pharmacol 35(24): 4493-4497.

21. Yardley JP, Husbands GE, Stack G, Butch J, Bicksler J, et al. (1990) 2-Phenyl-2-(1-hydroxycycloalkyl)ethylamine derivatives: synthesis and antidepressant activity. J Med Chem 33(10): 2899-2905.

22. Bymaster FP, Dreshfield-Ahmad LJ, Threlkeld PG, Shaw JL, Thompson $\mathrm{L}$, et al. (2001) Comparative affinity of duloxetine and venlafaxine for serotonin and norepinephrine transporters in vitro and in vivo, human serotonin receptor subtypes, and other neuronal receptors. Neuropsychopharmacol 25(6): 871-880.

23. Meanwell NA (2011) Synopsis of some recent tactical application of bioisosteres in drug design. J Med Chem 54(8): 2529-2591.

24. Tung R (2009) Heavyweight drugs. Drug discovery; Chemistry \& Industry pp 24-26.

25. Tung, R (2010) The development of deuterium-containing compounds. Innovations Pharm Tech Discov Tech pp 24-28.

26. Birds Pharma AG: BDD-10103; A 3rd generation 'perisone' and an appropriate formulation for improved treatment of acute back pain and spasticity.

27. Nelson SD, Trager WF (2003) The use of deuterium isotope effects to probe the active site properties, mechanism of cytochrome P450catalyzed reactions, and mechanisms of metabolically dependent toxicity. Drug Metab Dispos 31(12): 1481-1498.

28. Keefer LK, Lijinsky W, Garcia H (1973) Deuterium isotope effect on the carcinogenicity of dimethylnitrosamine in rat liver. J Natl Cancer Inst 51(1): 299-302.

29. Swann PF, Mace R, Angeles RM, Keefer LK (1983) Deuterium isotope effect on metabolism of $\mathrm{N}$-nitrosodimethylamine in vivo in rat. Carcinogenesis 4(7): 821-825.

30. Streeter AJ, Nims RW, Sheffels PR, Heur YH, Yang CS, et al. (1990) Metabolic denitrosation of $\mathrm{N}$-nitrosodimethylamine in vivo in the rat. Cancer Res 50(4): 1144-1150.

31. Elespuru RK (1987) Deuterium isotope effect in mutagenesis by nitroso compounds. Mutat Res 54(4): 265-270.

32. Thust R, Mendel J, Bach B, Schwarz H (1985) Detection of a deuterium isotope effect in di- and trisubstituted alkylphenylnitrosoureas: an SCE study in Chinese hamster V79-E cells. Carcinogenesis 6(1985): 873876.

33. Lijinsky W, Saavedra JE, Reuber MD, Singer SS (1982) Esophageal carcinogenesis in F344 rats by Nitrosomethylethylamines substituted in the ethyl group. J Natl Cancer Inst 68(4): 681-684. 
34. Brundrett RB, Cowens JW, Colvin M (1976) Chemistry of nitrosoureas: decomposition of deuterated 1, 3-bis(2-chlorethyl)-1-nitrosourea. J Med Chem 19(7): 958-961.

35. Weinkam RJ, Lin HS (1979) Reactions of 1,3-bis(2-chloroethyl)-1nitrosourea and 1-(2-chloroethyl)-3-cyclohexyl-1-nitrosourea in aqueous solution. J Med Chem 22(10): 1193-1198.

36. Moloney SJ, Prough, RA (1983) Studies on the pathway of methane formation from procarbazine, a 2-methylbenzylhydrazine derivative, by rat liver microsomes. Arch Biochem Biophys 221(2): 577-584.

37. Prough RA, Brown MI, Dannan GA, Guengerich FP (1984) Major isozymes of rat liver microsomal cytochrome P-450 involved in the $\mathrm{N}$-oxidation of $\mathrm{N}$-isopropyl-alpha-(2-methylazo)-p-toluamide, the azo derivative of procarbazine. Cancer Res 44(2): 543-548.

38. Chapman RM (1984) Effect of cytotoxic therapy on sexuality and gonadal function. In: Perry MC, Yarbro J W (Edn)Toxicity of Chemotherapy; Grune and Stratton. New York pp343-363.

39. Yost GS, Horstman MG, el Walily AF, Gordon WP, Nelson SD (1985) Procarbazine spermatogenesis toxicity: deuterium isotope effects point to regioselective metabolism in mice. Toxicol Appl Pharmacol 80(2): 316-322.

40. Holme JA, Søderlund EJ, Brunborg G, Hongslo JK, Trygg B, et al. (1989) Comparative genotoxicities of procarbazine and two deuterated analogs in mammalian cells in vitro and in vivo. Mutagenesis 4(5): 355-360.

41. Marques MM, Beland FA (1997) Identification of tamoxifenDNA adducts formed by 4-hydroxytamoxifen quinone methide. Carcinogenesis 18(10): 1949-1954.

42. Phillips DH, Potter GA, Horton MN, Hewer A, Crofton-Sleigh C, et al. (1994) Reduced genotoxicity of [D3-ethyl]-tamoxifen implicates $\alpha$-hydroxylation of the ethyl group as a major pathway of tamoxifen activation to a liver carcinogen. Carcinogenesis 15: 1487-1492.

43. Gad SC (2014) Phenacetin. Encyclopedia of Toxicology 3: 862-864.

44. Nelson SD, Garland WA, Mitchell JR, Vaishnav Y, Statham CN, et al. (1978) Deuterium isotope effects on the metabolism and toxicity of phenacetin in hamsters. Drug Metab Dispos 6(4): 363-367.

45. Yun CH, Miller GP, Guengerich FP (2000) Rate-determining steps in phenacetin oxidations by human cytochrome $\mathrm{P}_{450} 1 \mathrm{~A} 2$ and selected mutants. Biochemistry 39(37): 11319-11329.

46. Büch H, Buzello W, Heymann E, Krisch K (1969) Inhibition of phenacetin- and acetanilide induced methemoglobinemia in the rat by the carboxylesterase inhibitor bis-[p-nitrophenyl] phosphate. Biochem Pharmacol 18(4): 801-811.

Your next submission with Juniper Publishers will reach you the below assets

- Quality Editorial service

- Swift Peer Review

- Reprints availability

- E-prints Service

- Manuscript Podcast for convenient understanding

- Global attainment for your research

- Manuscript accessibility in different formats ( Pdf, E-pub, Full Text, Audio)

- Unceasing customer service

Track the below URL for one-step submission https://juniperpublishers.com/online-submission.php 